\title{
Mutação G20210A no gene da protrombina, fator V de Leiden e anticorpos anticardiolipina não influenciam a sobrevida do enxerto renal após o transplante
}

Prothrombin G20210A gene mutation, factor V Leiden and anticardiolipin antibodies do not influence renal graft survival after transplantation

\section{Autores}

Luis Klaus A. da Rocha ${ }^{1}$

Nelson Zocoler

Galante ${ }^{2}$

Vivian Angélica

Castilho Alvarez ${ }^{5}$

Kelsy C. N. Areco ${ }^{4}$

Maria Aparecida E. Noguti ${ }^{1}$

Rogério Q. Amaral ${ }^{3}$

Luis Eduardo C.

Andrade ${ }^{3}$

Clóvis Araujo Peres ${ }^{4}$

José O. Medina

Pestana ${ }^{2}$

Dayse M. Lourenço ${ }^{1}$

'Departamento de Medicina, Disciplina de Hematologia - UNIFESP - São Paulo, SP, Brasil

${ }^{2}$ Departamento de Medicina, Disciplina de Nefrologia - UNIFESP - São Paulo, SP,

Brasil

${ }^{3}$ Departameto de Medicina, Disciplina de Reumatologia - UNIFESP - São Paulo, SP, Brasil

${ }^{4}$ Departamento de Medicina Preventiva, Disciplina de Bioestatística - UNIFESP São Paulo, SP, Brasil

${ }^{5}$ Departamento de Tecnologia Medica de la Universidad de Antofagasta, Chile.

Data de submissão: 21/07/2009 Data de aprovação: 07/10/2009

Correspondência para: José O. Medina Pestana Hospital do Rim e Hipertensão Rua Borges Lagoa, número 960, $11^{\circ}$ andar Vila Clementino - São Paulo - Brasil

CEP: 04038-002.

Tel: (11) 5087-8056

Fax: (11) 5087-8008

E-mail: medina@hrim.com.br

Declaramos a inexistência de conflitos de interesse.

\section{Resumo}

Introdução: Complicações tromboembólicas são importantes fatores de risco para perda do enxerto e pior evolução após o transplante renal. Pacientes com defeito trombofílico apresentam maior risco de complicações tromboembólicas. Foram analisados, entre receptores de transplante renal, a prevalência de defeito trombofílico e o risco atribuído a esta condição para a perda do enxerto e para o desenvolvimento de tromboses intravasculares. Métodos: Estudo do tipo coorte incluindo 388 receptores adultos analisados quanto à presença de trombofilia de acordo com a pesquisa de anticorpos anticardiolipina (aCL) por ELISA e das mutações G1691A no gene do fator V (FV) e G20210A no gene da protrombina (PT) por PCR multiplex. Resultados: Defeito trombofílico foi identificado em $25,8 \%$ dos pacientes. As taxas de sobrevida de 2 anos do enxerto foram semelhantes entre os pacientes com e sem defeito trombofílico ( $94 \%$ versus $94 \%, p=0,53)$, bem como a sobrevida dos enxertos livres de tromboses intravasculares $(97 \%$ versus $97 \%$, $\mathrm{p}=0,83)$. Pacientes com defeito trombofílico apresentaram prevalência de tromboses intravasculares semelhante à do grupo-controle $(3 \%$ versus $3,5 \%, \mathrm{p}=0,82)$. O transplante renal anterior foi associado a maior risco de perda de enxerto (OR 20,8, p < 0,001) e de ocorrência de tromboses intravasculares ( $\mathrm{OR}$ $6,8, \mathrm{p}=0,008)$. Conclusões: As prevalências das mutações FV G1691A e PT G20210A na população estudada foram semelhantes às $\mathrm{da}$ população geral não transplantada, e a prevalência de anticorpos aCL superou a observada entre os indivíduos sadios. Não houve associação entre os marcadores de trombofilia estudados e a sobrevida em médio prazo do transplante renal.

Palavras-chave: trombofilia, anticorpos anticardiolipina, fator $\mathrm{V}$ de Leiden, mutação G20210A no gene da protrombina, transplante renal, rejeição de enxerto, análise de sobrevida, modelos logísticos.

[J Bras Nefrol 2009;31(4):277-285] EElsevier Editora Ltda.

\section{Abstract}

Introduction: Thromboembolic complications are important risk factors for graft failure and worse renal transplantation outcome. Patients with thrombophilic disorders have a higher risk of thromboembolic complications. The prevalence of thrombophilic disorders and the associated risk for graft failure and for intravascular thrombosis were analyzed in renal transplant recipients. Methods: This is a cohort study of 388 adult recipients investigated regarding the presence of thrombophilia, through the search for anticardiolipin antibodies (aCL) via ELISA and FV G1691A and PT G20210A gene mutations by multiplex PCR. Results: Thrombophilic disorders were identified in $25.8 \%$ of the patients. The 2-year graft survival was similar among patients with and without thrombophilic disorder $(94 \%$ versus $94 \%, \mathrm{p}=0.53)$, and so was the survival free of intravascular thrombosis $(97 \%$ versus $97 \%$, $\mathrm{p}=0.83)$. The prevalence of intravascular thrombosis was similar in both groups $(3 \%$ versus $3.5 \%, p=0.82$ ). Patients with previous kidney transplantation had a higher risk of graft failure (OR 20.8, p < 0.001) and of intravascular thrombosis (OR 6.8, $\mathrm{p}=0.008$ ). Conclusions: The prevalences of FV G1691A and PT G20210A gene mutations in this cohort of patients were similar to those of the general non-transplanted population. The prevalence of aCL antibodies was higher in this cohort than that observed in healthy individuals. The thrombophilic markers studied did not predict the medium-term survival of renal transplant.

Keywords: thrombophilia, anticardiolipin antibodies, factor V Leiden mutation, prothrombin G20210A mutation, renal transplantation, graft rejection, survival analysis, logistic models. 


\section{INTRODUÇÃO}

A disfunção aguda do enxerto é uma complicação frequente após o transplante renal. Nela, estão envolvidos processos fisiopatológicos de origem imunológica e não imunológica. Maior atenção tem sido voltada, nos últimos anos, à prevenção e ao controle dos eventos de natureza não imunológica, uma vez que o controle da resposta imune atualmente é efetivo e acompanhado de baixa incidência de disfunção aguda do enxerto atribuída a eventos imunológicos. A trombofilia é um importante fator de risco não imunológico para a evolução do enxerto durante o primeiro ano após o transplante renal ${ }^{1}$. Caracteriza-se por uma tendência aumentada para a formação de trombos e pode ser classificada em primária (genética) ou secundária (adquiria).2, 3

O número de condições associadas à trombofilia aumentou proporcionalmente ao desenvolvimento de novas metodologias que permitiram a identificação de maior número de polimorfismos em genes relacionados com o sistema de coagulação e de anticoagulação. A mutação G1691A no gene do fator V (FV) do sistema de coagulação (Fator V de Leiden), ${ }^{4}$ a mutação G20210A no gene da protrombina (PT) e a presença de anticorpos anticardiolipina $(\mathrm{aCL})^{6,7}$ circulantes no sangue periférico são importantes condições associadas à trombofilia e podem influenciar o risco de perda do enxerto renal atribuída a eventos trombóticos. Estudos populacionais indicam, entretanto, que a prevalência de trombofilia não é uniforme. Embora as mutações FV G1691A e PT G20210A sejam altamente prevalentes na população caucasiana, menor proporção de indivíduos de origem negra africana $^{8}$ e brasileira ${ }^{9,10}$ apresenta essas mutações. Por outro lado, a identificação de anticorpos aCL, uma condição relacionada com o defeito trombofílico adquirido, não apresenta qualquer correlação com a etnia. ${ }^{11}$ Neste estudo, descrevemos as prevalências e a influência destas alterações na sobrevida do enxerto em uma população brasileira de receptores de transplante renal.

\section{Material e Métodos}

\section{PACIENTES}

O estudo incluiu 388 receptores de transplantes renais realizados no Hospital do Rim e Hipertensão - Universidade Federal de São Paulo (UNIFESP), no período de $1^{\circ}$ de maio de 2001 a 31 de julho de 2002, e acompanhados há pelo menos 1 ano após o transplante. Foram excluídos os pacientes com idade inferior a 18 anos na data do transplante e os que não concordaram em participar espontaneamente do estudo. O projeto de pesquisa foi previamente analisado e aprovado pelo Comitê de Ética em Pesquisa da UNIFESP (Protocolo no 0628/06). Todos os pacientes assinaram o Termo de Consentimento Livre e Esclarecido.

Os pacientes receberam imunossupressão tríplice com um inibidor de calcineurina (ciclosporina ou tacrolimo), prednisona e uma droga adjuvante (azatioprina, micofenolato mofetil ou sirolimo) e foram acompanhados no período pós-transplante em conformidade com os procedimentos padronizados na UNIFESP. ${ }^{12}$

\section{DesenHo do ESTUdO E VARIÁVEIS ANALISADAS}

O estudo foi de tipo coorte e analisou a prevalência e o risco atribuídos ao defeito trombofílico para o desenvolvimento de tromboses intravasculares entre receptores de transplante renal. O defeito trombofílico foi definido como a identificação de um ou mais dos seguintes marcadores em um único paciente: anticorpos aCL da classe IgM ou IgG no sangue periférico, mutação G1691A no gene do Fator V (Fator V de Leiden) e mutação G20210A no gene da protrombina. As informações clínicas foram obtidas dos prontuários médicos até 31 de outubro de 2004. Foram analisadas as incidências de tromboses da artéria e da veia renal do enxerto e também as de vasos de outras localizações. O diagnóstico de trombose intravascular foi sempre confirmado por meio de Dopplerfluxometria ou angiografia do vaso indicado após suspeita clínica. A avaliação histológica indicativa de trombose de vasos intrarrenais também foi utilizada como critério para o diagnóstico de trombose intravascular. Os desfechos clínicos analisados foram a sobrevida do enxerto e a sobrevida do enxerto livre de trombose intravascular entre os pacientes com e sem defeito trombofílico. A perda do enxerto renal foi definida como o retorno do paciente ao programa regular de diálise ou o óbito do paciente com o rim em funcionamento.

As características demográficas estudadas foram a idade no momento do transplante, o sexo, a causa da insuficiência renal crônica, o tipo de doador, o tempo em diálise, o tipo de diálise e o número de transplantes realizados anteriormente.

\section{ANÁLISES LABORATORIAIS}

Uma amostra de $10 \mathrm{~mL}$ de sangue periférico foi obtida de cada paciente em tempos variados após a data do transplante durante o período compreendido entre $1^{\circ}$ de março de 2003 e 31 de março de 2004. Cada amostra foi divida em duas alíquotas de iguais volumes; uma foi mantida em tubo plástico seco e outra, 
em tubo plástico contendo EDTA, de modo a permitir o armazenamento, respectivamente, de soro e sangue total separadamente a $-70^{\circ} \mathrm{C}$.

\section{DETERMINAÇÃO DE ANTICORPOS ANTICARDIOLIPINA}

As amostras de soro foram testadas quanto à presença de anticorpos de classe IgG e IgM específicos para cardiolipina por meio da técnica de ELISA (EnzymeLinked Immunosorbent Assay). O antígeno cardiolipina (Sigma ${ }^{\circledR}$ C1649, EUA) foi previamente diluído a $50 \mu \mathrm{g} / \mathrm{mL}$ com etanol gelado, e $50 \mu \mathrm{L}$ desta solução foram adicionados a cada um dos poços de uma placa de 96 poços com fundos planos (Nunc ${ }^{\circledR}$, EUA). A placa foi mantida em incubação noturna a $4^{\circ} \mathrm{C}$ até o dia seguinte para a completa evaporação do solvente. Em seguida, a placa foi incubada com PBS contendo soro bovino adulto a $10 \%$ (PBS-BSA) durante 1 hora, em temperatura ambiente, e, posteriormente, lavada por três vezes com Tween-PBS. Cem microlitros de soro a ser testado, diluídos à razão de 1:50 com PBS-BSA, foram adicionados a cada um dos poços contendo o antígeno cardiolipina e também aos poços-controle negativos. Reações-controle positivas foram preparadas utilizando-se diluições 1/50, 1/100, 1/200 e 1/400 dos padrões IgM e IgG humanos (Louisville APL diagnostics $^{\circledR}$, EUA). A placa foi submetida a mais uma incubação noturna a $4^{\circ} \mathrm{C}$ até o dia seguinte e, em seguida, foi lavada três vezes com Tween-PBS. Anticorpos de cabra anti-IgG ou anti-IgM humanos (Calbiochem Laboratories, La Jolla, CA) marcados com fosfatase alcalina (Calbiochem ${ }^{\circledR}$, EUA) foram diluídos à razão de 1:4.000 em PBS-BSA e $100 \mu \mathrm{L}$ desta solução foram adicionados a cada um dos poços. Após a placa ser submetida à incubação por 1 hora à temperatura ambiente e lavada três vezes com Tween-PBS, $100 \mu \mathrm{L}$ de solução substrato contendo p-nitrofenil-fosfato a $1 \mathrm{mg} / \mathrm{mL}$ em tampão dietanolamina $1 \mathrm{M}$ e $\mathrm{MgCl}_{2} 0,5$ $\mathrm{mM}(\mathrm{pH} 9,8)$ foram adicionados a cada poço. A placa foi, então, mantida no escuro por 30 minutos à temperatura ambiente, e a reação foi posteriormente interrompida mediante adição de $20 \mu \mathrm{L}$ de hidróxido de sódio $1,5 \mathrm{~N}$. O cromóforo gerado foi quantificado por absorbância a $405 \mathrm{~nm}$.

\section{Genotipagem}

A identificação das mutações FV G1691A e PT G20210A foi feita utilizando-se DNA de amostras de sangue total. Os primers 5' CTTGAAGGAAATGCCCCATTA - 3' (sense), 5' TGCCCAGTGCTTAACAAGACCA - 3' (antisense), 5' - TCTAGAAACAGTTGCCTGGC - 3' (sense) e
5' - ATAGCACTGGGAGCATTGAAGC - 3' (antissense) foram utilizados para amplificação simultânea, respectivamente, dos genes do FV e da PT pela técnica de PCR (polymerase chain reaction) multiplex. Os produtos gerados após a amplificação foram submetidos à digestão enzimática combinada ( $\mathrm{Mnl}$ I e Hind III) e, posteriormente, separados por eletroforese em gel de agarose, de acordo com a metodologia estabelecida por Gómez et al..$^{13}$ Segundo estes autores, os produtos de amplificação do FV geram fragmentos de 67 e 153 pares de base (pb) quando a mutação G1691A está presente, e fragmentos de 37, 67 e 116 pb quando ambos os alelos não apresentam esta mutação. Os produtos de amplificação do PT geram fragmentos de $15,23,58$ e 249 pb quando a mutação G20210A está presente, e fragmentos de 15,58 e 272 pb quando ambos os alelos não apresentam esta mutação. A identificação de fragmentos de 116, 153, 272 e 249 pb em uma mesma amostra é indicativa da presença de ambas as mutações simultaneamente.

\section{ANÁLISE ESTATÍSTICA}

As variáveis numéricas foram descritas por meio de médias e de desvios-padrão, e as variáveis categóricas, por meio de frequências percentuais. As variáveis categóricas relacionadas com as características demográficas dos receptores, às prevalências dos diversos marcadores relacionados com defeito trombofílico, de tromboses da artéria e da veia renal do enxerto e de tromboses de vasos em outras localizações foram comparadas entre os dois grupos, utilizando-se o teste do qui-quadrado ou o teste exato de Fisher, quando apropriado. As variáveis numéricas foram comparadas pelo teste $t$ de Student. O método de Kaplan-Meier foi utilizado para analisar a sobrevida do enxerto e a sobrevida do enxerto livre de trombose intravascular. A comparação entre os grupos foi feita por meio do teste $\log$ Rank. Foi utilizada análise de regressão logística múltipla para determinar a contribuição das variáveis idade, sexo, realização prévia de pelo menos um transplante e presença de defeito trombofílico sobre o risco de perda do enxerto e de ocorrências de tromboses intravasculares após o transplante. Um valor de $\mathrm{p}<0,05$ foi considerado estatisticamente significativo. Todas as análises foram realizadas com o programa SPSS (versão 7.5.1, SPSS Inc., Chicago, IL, USA, 1996).

\section{Resultados}

O defeito trombofílico foi identificado em $100(25,8 \%)$ pacientes. As prevalências do defeito trombofílico, de 
acordo com a identificação de anticorpos anticardiolipina e a pesquisa de mutações nos genes do FV e PT, são mostradas na Tabela 1. A presença de anticorpos aCL no sangue periférico foi o principal marcador de trombofilia observado na população estudada - 46 pacientes $(11,8 \%)$ apresentaram anticorpos aCL da classe $\operatorname{IgM}, 38(9,8 \%)$ da classe IgG e $5(1,3 \%)$ de ambas as classes -, seguida pelas mutações PT G20210A - 13 pacientes $(3,35 \%)$ - e FV G1691A - seis pacientes (1,5\%). A prevalência de defeito trombofílico associado à identificação de mais de um dos marcadores estudados foi igual ou inferior a $1 \%$ (Tabela 1 ).

As características demográficas dos pacientes são apresentadas na Tabela 2. Os pacientes com e sem defeito trombofílico não apresentaram diferenças quanto à idade em anos $(38,2 \pm 11,6$ versus $38,8 \pm 11,4$, $\mathrm{p}=0,6)$, ao sexo $(53 \%$ do sexo masculino versus $58,7 \%, p=0,3)$, às causas da insuficiência renal crônica (glomerulonefrite crônica $20 \%$ versus $20,8 \%$, $\mathrm{p}=0,8$; hipertensão arterial sistêmica $15 \%$ versus $17 \%, p=0,6$; rins policísticos $6 \%$ versus $4,9 \%$, $\mathrm{p}=0,6$; diabetes mellitus $6 \%$ versus $4,5 \%, \mathrm{p}=0,6$; lúpus eritematoso sistêmico $5 \%$ versus $3,1 \%, p=0,4$; pielonefrite crônica $0 \%$ versus $2,1 \%, \mathrm{p}=0,3$; e outras etiologias $8 \%$ versus $5,9 \%, \mathrm{p}=0,5)$, ao tipo de doador ( $72 \%$ receberam rins de doadores vivos versus $72,6 \%$, $\mathrm{p}=0,9)$, ao tempo de diálise em meses $(40,9 \pm 29,8$ versus $33,6 \pm 37,1, \mathrm{p}=0,17$ ) e ao número de transplantes realizados anteriormente por um único doador $196 \%$ dos receptores receberam um transplante renal pela primeira vez versus $94,1 \%, \mathrm{p}=0,5)$.

Entretanto, os pacientes sem defeito trombofílico haviam recebido tratamento com hemodiálise antes do transplante com frequência significativamente menor do que os pacientes com defeito trombofílico ( $88 \%$ versus $95,8 \%, p=0,02)$.

\section{Anticorpos ACL}

As distribuições dos pacientes, de acordo com a pesquisa de anticorpos aCL, tanto da classe IgM quanto da classe IgG, no sangue periférico em função do tempo após o transplante em que as amostras de sangue foram colhidas, é mostrada na Figura 1. A maioria $(98,8 \%)$ das amostras positivas para anticorpos aCL foi obtida de pacientes acompanhados há, pelo menos, 1 ano após o transplante. Anticorpos aCL não foram identificados nas amostras obtidas de pacientes em seguimento de até 10 meses. As concentrações séricas dos anticorpos aCL da classe IgG e IgM em função do tempo de acompanhamento após o transplante são mostradas na Figura 2.

\begin{tabular}{|c|c|c|}
\hline \multicolumn{3}{|c|}{$\begin{array}{l}\text { PREVALÊNCIA DO DEFEITO TROMBOFílICO NA POPULAÇÃO ESTUDADA DE ACORDO COM A IDENTIFICAÇÃO DE } \\
\text { ANTICORPOS SÉRICOS ANTICARDIOLIPINA DA CLASSE IGM OU IGG, MUTAÇĀO FV G1691A E MUTAÇÃO PT G20210A }\end{array}$} \\
\hline Defeito Trombofílico & $\mathrm{n}$ & Prevalência \\
\hline $\mathrm{aCL} \lg \mathrm{M}$ & 42 & $10,8 \%$ \\
\hline $\mathrm{aCL} \operatorname{IgM}$ e aCL IgG & 4 & $1,0 \%$ \\
\hline $\mathrm{aCL} \lg M, \mathrm{aCL} \lg \mathrm{G}$ e FV G1691A & 0 & $0,0 \%$ \\
\hline aCL IgM, aCL IgG e PT G20210A & 1 & $0,3 \%$ \\
\hline aCL IgM e FV G1691A & 0 & $0,0 \%$ \\
\hline aCL IgM, FV G1691A e PT G20210A & 0 & $0,0 \%$ \\
\hline aCL IgM e PT G20210A & 4 & $1,0 \%$ \\
\hline $\mathrm{aCL} \lg \mathrm{G}$ & 35 & $9,0 \%$ \\
\hline aCL IgG e FV G1691A & 2 & $0,5 \%$ \\
\hline aCL IgG, FV G1691A e PT G20210A & 0 & $0,0 \%$ \\
\hline aCL IgG e PT G20210A & 1 & $0,3 \%$ \\
\hline FV G1691A & 4 & $1,0 \%$ \\
\hline FV G1691A e PT G20210A & 0 & $0,0 \%$ \\
\hline PT G20210A & 7 & $1,8 \%$ \\
\hline aCL IgG, aCL IgM, FV G1691A e PT G20210A & 0 & $0,0 \%$ \\
\hline Total & 100 & $25,8 \%$ \\
\hline
\end{tabular}

aCL IgM, anticorpo anticardiolipina da classe IgM; aCL lgG, anticorpo anticardiolipina da classe lgG; FV G1691A, mutação G1691A no gene do fator V (Fator V de Leiden); PT G20210A, mutação G20210A no gene da protrombina. 


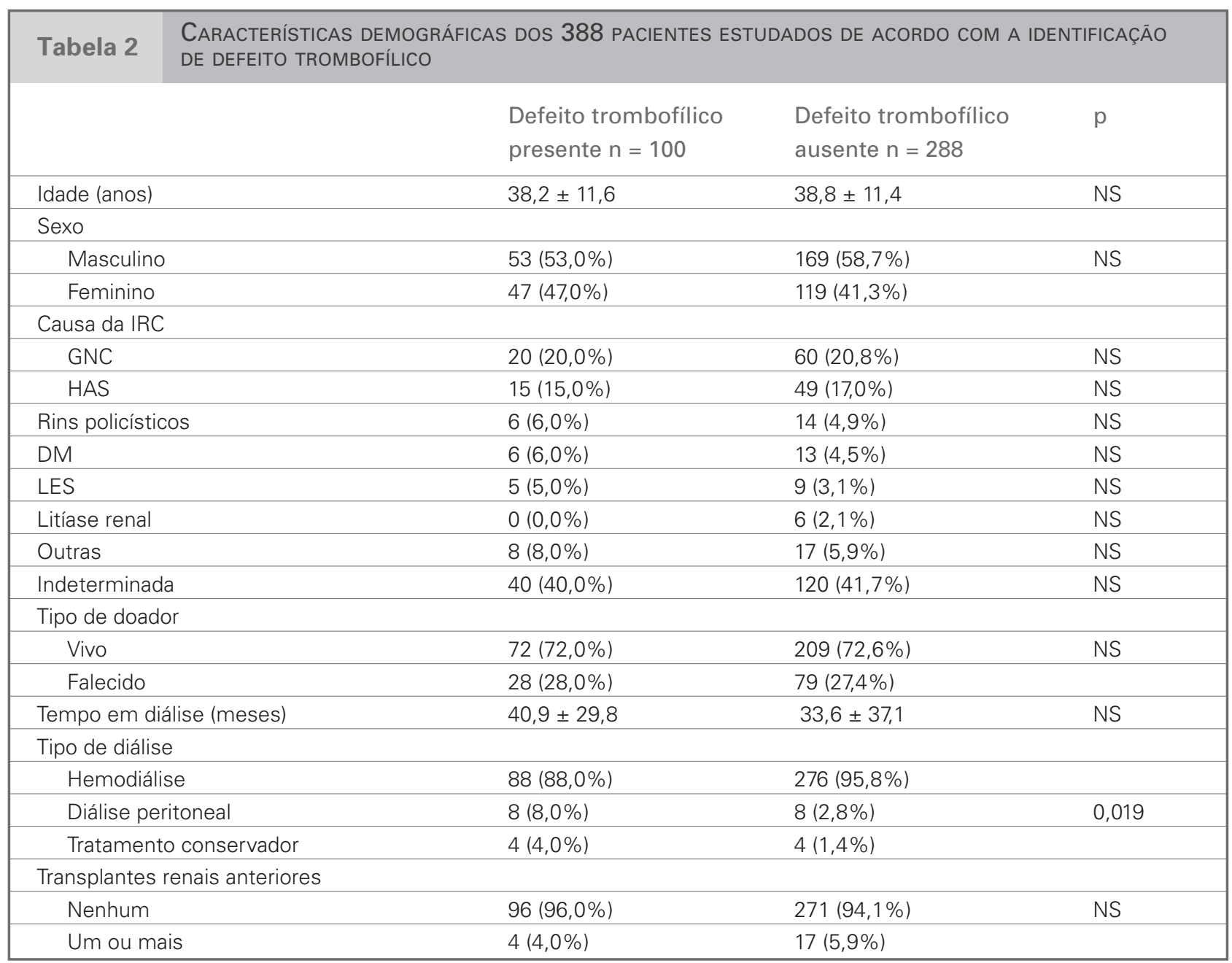

IRC, insuficiência renal crônica; GNC, glomerulonefrite crônica; HAS, hipertensão arterial sistêmica; DM, diabetes mellitus pré-transplante; LES, lúpus eritematoso sistêmico; NS, não significante.

Figura 1. Número de pacientes com amostras positivas e negativas para a pesquisa de anticorpos $\mathrm{aCL}$, tanto da classe $\lg M$ quanto $\lg G$, no sangue periférico em função do tempo após o transplante.

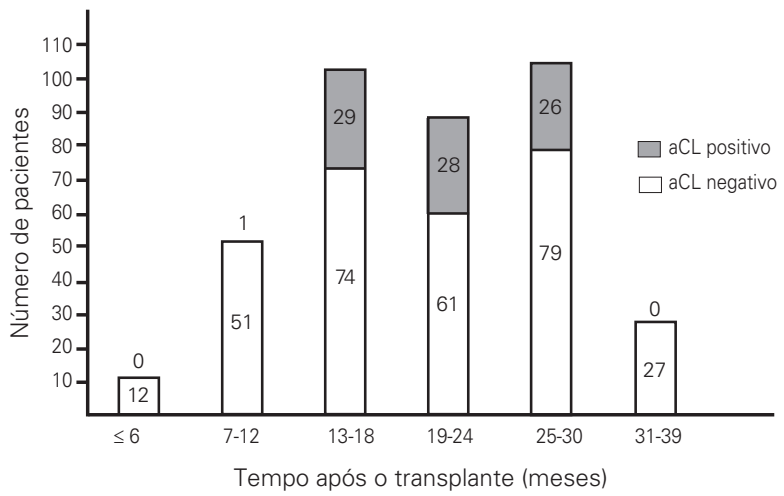

Figura 2. Concentrações séricas dos anticorpos aCL da classe IgG e IgM em função do tempo de acompanhamento após o transplante.

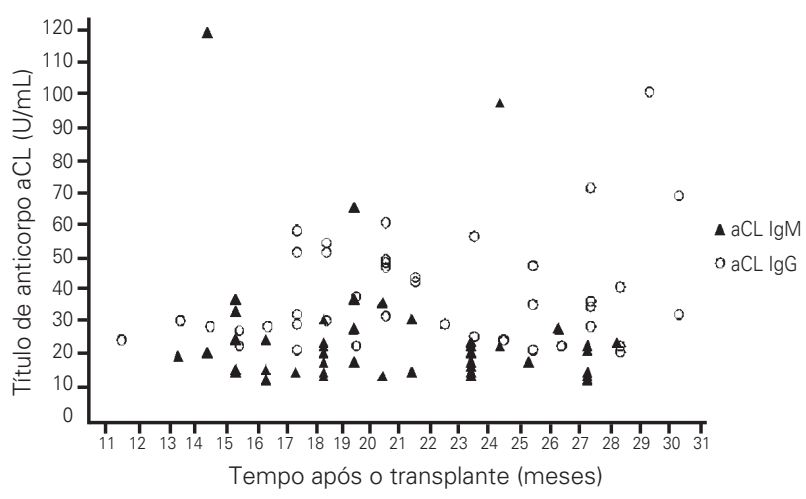




\section{ANÁLISES DE SOBREVIDA}

As curvas de sobrevida do enxerto incluindo todas as perdas e do enxerto livre de tromboses intravasculares são mostradas na Figura 3. Durante o período de seguimento, seis pacientes com trombofilia perderam o enxerto, três $(50 \%)$ em razão da rejeição aguda, dois $(33,3 \%)$ por trombose de artéria renal e um $(16,7 \%)$ devido a não função primária do enxerto.

Entre os pacientes sem trombofilia, 13 perderam o enxerto, seis $(46,1 \%)$ em razão da rejeição aguda, dois $(15,4 \%)$ por nefropatia crônica do enxerto, um $(7,7 \%)$ devido à trombose de artéria renal, um $(7,7 \%)$ em virtude de trombose de veia renal, um $(7,7 \%)$ devido ao óbito do paciente em razão de causa infecciosa e dois $(15,4 \%)$ devido a outras causas.

Os pacientes com defeito trombofílico apresentaram taxas de sobrevida do enxerto 1 e 2 anos após o transplante semelhantes às apresentadas pelos pacientes sem defeito trombofílico: $96,5 \%$ e $94 \%$ versus $95,8 \%$ e $94 \%$, respectivamente, $\mathrm{p}=0,53$.

Figura 3. Curvas de sobrevida do enxerto incluindo todas as perdas (A) e sobrevida do enxerto livre de tromboses em grandes vasos renais (B) entre receptores de transplante renal com e sem defeito trombofílico.

A
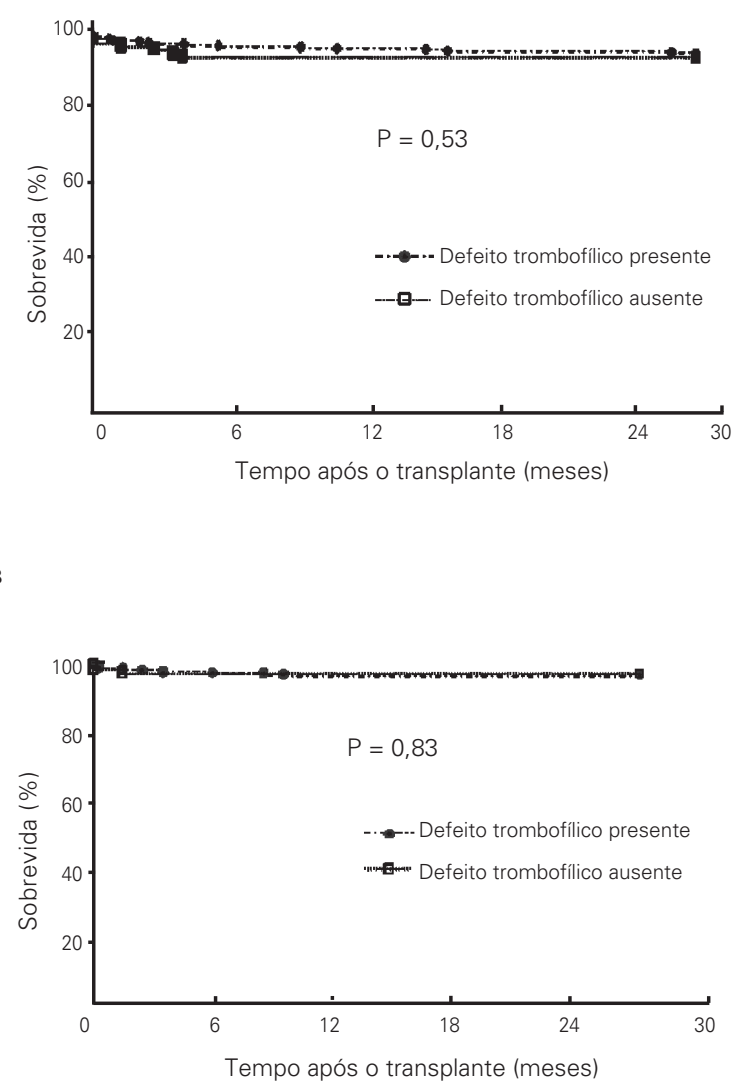

Os receptores com defeito trombofílico apresentaram incidência semelhante de trombose de um dos grandes vasos renais com relação aos pacientes sem defeito trombofilico - dois pacientes $(2 \%)$ versus quatro pacientes $(1,4 \%)$, respectivamente, $\mathrm{p}=0,67$. Esses eventos de trombose resultaram em perda do enxerto renal em ambos os pacientes com defeito trombofílico e em dois pacientes sem defeito trombofílico. Trombectomia cirúrgica e fibrinólise enzimática com estreptoquinase, aplicadas individualmente, foram eficazes para prevenir a perda do enxerto renal nos demais pacientes sem defeito trombofílico que apresentaram trombose em um dos grandes vasos renais.

As sobrevidas dos enxertos livres de tromboses intravasculares 1 e 2 anos após o transplante foram de $96,5 \%$ e $97 \%$ versus $96,5 \%$ e $97 \%$, respectivamente, entre os pacientes sem e com defeito trombofílico, $\mathrm{p}$ $=0,83$ (Figura 3$)$.

\section{COMPLICAÇÕES APÓS O TRANSPLANTE}

A prevalência de tromboses vasculares na população estudada foi de $3,3 \%$. Os pacientes portadores de defeito trombofílico apresentaram prevalência de tromboses vasculares semelhante à do grupo de pacientes que não apresentavam defeito trombofílico - três pacientes $(3 \%)$ versus dez pacientes $(3,5 \%), p=0,82$. Os territórios vasculares em que foram observados eventos trombóticos nos pacientes portadores de defeito trombofílico foram a artéria renal do enxerto em um paciente, a veia renal do enxerto em um paciente e veias profundas do membro inferior direito em um paciente.

Entre os pacientes sem defeito trombofílico, os territórios vasculares em que foram observados eventos trombóticos foram a artéria renal do enxerto em um paciente, a veia renal do enxerto em dois pacientes, veias profundas do membro inferior direito em dois pacientes, veia subclávia direita em um paciente, veia subclávia esquerda e plexo hemorroidário em um paciente, veia jugular interna direita em um paciente, veias profundas do membro inferior esquerdo em um paciente, e artéria renal do enxerto e veias profundas do membro inferior direito em um paciente.

Os fatores de risco e os respectivos odds ratios $(\mathrm{OR})$ obtidos pela análise multivariada para a perda do enxerto e para a ocorrência de tromboses vasculares são mostrados na Tabela 3.

Pacientes previamente submetidos ao transplante renal apresentaram maior risco de perda de enxerto (OR $20,8, p<0,001)$ e de ocorrência de tromboses vasculares (OR 6,8, $\mathrm{p}=0,008)$. O sexo, a idade e a presença de trombofilia não apresentaram correlação com o risco de perda do enxerto ou de ocorrência de tromboses vasculares (Tabela 3). 
Tabela 3 FATORES DE RISCO PARA A PERDA DO ENXERTO E PARA A OCORRÊNCIA DE TROMBOSES VASCULARES APÓS O TRANSPLANTE RENAL

\begin{tabular}{|c|c|c|c|c|}
\hline & \multicolumn{2}{|c|}{ Perda do enxerto } & \multicolumn{2}{|c|}{ Tromboses vasculares } \\
\hline & OR (IC 95\%) & $\mathrm{p}$ & OR (IC 95\%) & $\mathrm{p}$ \\
\hline Idade $\leq 38$ anos e 6 meses & $1,00(0,95-1,04)$ & 0,866 & $1,03(0,98-1,08)$ & 0,229 \\
\hline Sexo feminino & $1,04(0,38-2,89)$ & 0,940 & $0,95(0,30-3,05)$ & 0,935 \\
\hline Transplante anterior & $20,8(6,95-62,72)$ & $<0,001$ & $6,8(1,65-27,91)$ & 0,008 \\
\hline Trombofilia & $1,68(0,57-4,97)$ & 0,348 & $0,93(0,25-3,52)$ & 0,918 \\
\hline
\end{tabular}

OR, odds ratio; IC, intervalo de confiança.

\section{Dıscussão}

A associação entre estado trombofílico e tromboses vasculares é bem estabelecida entre indivíduos geneticamente predispostos quando expostos a estímulos ambientais ou adquiridos, como gravidez, tabagismo, procedimentos cirúrgicos, neoplasias e uso de anticoncepcionais orais. ${ }^{14}$ Embora a trombofilia também predisponha a ocorrência de eventos trombóticos primários, ${ }^{15}$ sua associação a complicações trombóticas e pior evolução do enxerto após o transplante renal é ainda controversa.

Os anticorpos aCL e as mutações FV G1691A e PT G20210A foram utilizados neste estudo como marcadores de trombofilia. A prevalência de anticorpos aCL varia de acordo com a população estudada e com a metodologia laboratorial empregada para detectá-los. Aproximadamente $1 \%$ a $5 \%$ dos indivíduos jovens saudáveis apresentam anticorpos aCL, ${ }^{16}$ contrastando com frequências entre $12 \%$ e $30 \%$ observadas em pacientes com lúpus eritematoso sistêmico, ${ }^{17} 19 \%$ em pacientes renais crônicos aguardando o transplante renal, ${ }^{18} 28 \%$ em transplantados não lúpicos, ${ }^{7}$ e $57 \%$ em receptores de transplante renal em que o enxerto não apresentou função primária. ${ }^{6}$

Embora o significado clínico dos anticorpos aCL em muitos destes pacientes e indivíduos saudáveis seja desconhecido, estudos prospectivos indicam associação positiva entre anticorpos aCL e eventos de trombose venosa, ${ }^{19}$ infarto do miocárdio, ${ }^{20} \mathrm{e}$ acidente vascular cerebral. ${ }^{21}$

Neste estudo envolvendo uma coorte de 388 receptores de transplantes renais, a prevalência de anticorpos aCL, tanto de classe $\operatorname{IgM}$ quanto $\operatorname{IgG}$, foi de $22,9 \%$, mas não foi observada nenhuma associação ao risco de perda do enxerto ou de ocorrência de tromboses vasculares. Esses anticorpos foram identificados em amostras colhidas somente a partir do $10^{\circ}$ mês de acompanhamento após o transplante.
Embora tenha sido recentemente observado um efeito modulador diretamente atribuído à imunossupressão e ao próprio órgão transplantado sobre o concentração sérica de anticorpos aCL após o transplante renal, ${ }^{22}$ salientamos que a metodologia utilizada no presente estudo, incluindo apenas uma amostra de sangue por paciente, não permitiu estabelecer precisamente a cinética de desenvolvimento de anticorpos aCL em função do tempo após o transplante. Nenhum ajuste com relação ao tempo após o transplante para a coleta das amostras foi empregado, já que as amostras de sangue foram obtidas com a única finalidade de identificar pacientes portadores de defeito trombofílico de acordo com marcadores selecionados. Estas informações reforçam o conceito de que a influência deste fator de risco sobre os resultados do transplante renal é complexa e difícil de ser estabelecida.

A mutação FV G1691A é a disfunção trombofílica hereditária mais comumente encontrada, ocorrendo entre $5 \%$ e $8 \%$ na população geral, em $20 \%$ dos pacientes que apresentaram um episódio isolado de trombose e em até $50 \%$ dos pacientes com história pessoal ou familiar de trombose. ${ }^{1}$ A prevalência da mutação FV G1691A na população de receptores renais equivale à da população não transplantada, com pequenas variações de acordo com o grupo étnico estudado. ${ }^{4,23-25}$ No presente estudo, a prevalência desta mutação foi de $1,5 \%$, o que é equivalente à observada na população brasileira de não transplantados. ${ }^{9}$ A mutação PT G20210A, por sua vez, é a segunda disfunção trombofílica hereditária mais comum, com prevalência estimada de $2 \%$ a $3 \%$ tanto na população geral quanto na de receptores de transplante renal, ${ }^{5,23}$ sendo encontrada em $6 \%$ dos pacientes com um episódio isolado de trombose, e em $18 \%$ dos pacientes com episódios recorrentes de trombose. ${ }^{1}$ 
As prevalências de complicações trombóticas após o transplante renal entre os grupos de pacientes aqui estudados foram semelhantes. Apenas três pacientes portadores de defeito trombofílico apresentaram tromboses vasculares em vasos periféricos, todos com anticorpos aCL. Nenhum paciente com mutação FV G1691A ou PT G20210A apresentou complicações trombóticas. Embora os marcadores de trombofilia analisados tenham sido associados a complicações trombóticas e pior evolução após o transplante renal em estudos anteriores, ${ }^{4,5}$ outros fatores de risco influenciam significativamente a ocorrência de complicações trombóticas nestes pacientes. A insuficiência renal crônica é acompanhada de aumento de atividade do fator VII, do fibrinogênio, da geração de trombina ${ }^{26,27}$ além de prejuízo na função das plaquetas. ${ }^{28}$ Após o transplante renal, existe significativo declínio da atividade fibrinolítica ${ }^{29}$ e da ativação da proteína $\mathrm{C},{ }^{30}$ além de ativação de trombina, plaquetas e da via intrínseca da coagulação quando os pacientes estão imunossuprimidos com ciclosporina. ${ }^{29,31}$

As contribuições de cada um destes fatores de risco não foram analisadas no presente estudo e podem ter influenciado significativamente os resultados observados. Além disso, observamos que uma proporção significativamente maior de pacientes que não apresentavam defeito trombofílico recebeu tratamento com hemodiálise antes do transplante. $\mathrm{O}$ tratamento com hemodiálise é acompanhado por alterações estruturais e funcionais específicas nas plaquetas, ${ }^{32}$ podendo, também, ter contribuído para equiparar a prevalência das complicações trombóticas dos pacientes sem defeito trombofílico com a observada entre os pacientes que o apresentavam.

Tromboses primárias do enxerto ocorrem entre $1 \%$ e $7 \%$ dos receptores de transplante renal e podem representar até $25 \%$ das perdas dos enxertos ao final do primeiro ano de acompanhamento. ${ }^{33-36}$ Estas observações sugerem que a identificação de pacientes com risco aumentado de complicações trombóticas e a utilização de anticoagulação profilática podem beneficiar um número significativo de receptores de transplante renal. Contudo, a realização rotineira de testes de identificação da condição de trombofilia e a prescrição de anticoagulação profilática após o transplante renal são ainda assuntos controversos. Neste estudo prospectivo, ambos os grupos de receptores apresentaram sobrevida do enxerto 2 anos após o transplante de $94 \%$ e não houve diferença quanto às frequências de perdas de enxertos atribuídas a tromboses de vasos renais. O defeito trombofílico também não foi associado ao risco de perda do enxerto ou de ocorrência de tromboses vasculares após o transplante. Outros fatores de risco, e principalmente a exposição aos agentes imunossupressores, influenciam significativamente o risco de ocorrência de eventos trombóticos após o transplante renal e devem, também, ser considerados durante a triagem destes pacientes, visando à profilaxia destas complicações.

Outra observação importante foi a de que os receptores submetidos a um transplante renal anteriormente apresentaram maior risco de ocorrência de eventos trombóticos e de perda do enxerto. Estes achados indicam que medidas específicas devem ser aplicadas a estes pacientes buscando aperfeiçoar a sobrevida do enxerto. Irish et al., em 1997, observaram que muitos pacientes com perdas de enxertos renais secundárias a tromboses podem ser adequadamente submetidos a um novo transplante renal desde que recebam heparinização subcutânea profilática. ${ }^{24}$

Em conclusão, as prevalências das mutações FV G1691A e PT G20210A na população estudada foram semelhantes às da população geral não transplantada, e a prevalência de aCL superou a observada entre os indivíduos sadios. Não foi observada qualquer associação entre a presença destes marcadores de trombofilia e a evolução a médio prazo do transplante renal.

Os resultados deste estudo não subsidiam a utilização profilática de agentes anticoagulantes em receptores de transplante renal que apresentem anticorpos aCL, mutações FV G1691A ou PT G20210A sem associação a outros fatores de risco.

\section{REFERÊNCIAS}

1. Kujovich J. Thrombophilia and thrombotic problems in renal transplant patients. Transplantation 2004; 77:959-64.

2. Seligsohn U, Lubetsky A. Genetic susceptibility to venous thrombosis. N Engl J Med 2001; 344:1222-31.

3. Federman D, Kirsner R. An update on hypercoagulable disorders. Arch Intern Med 2001; 161:1051-6.

4. Wüthrich R, Cicvara-Muzar S, Booy C et al. Heterozygosity for the factor V Leiden (G1691A) mutation predisposes renal transplant recipients to thrombotic complications and graft loss. Transplantation 2001; 72:549-50.

5. Fischereder $\mathrm{M}$, Schneeberger $\mathrm{H}$, Lohse $\mathrm{P}$ et al. Increased rate of renal transplant failure in patients with the G20210A mutation of the prothrombin gene. Am J Kidney Dis 2001; 38:1061-4.

6. Wagenknecht D, Becker D, LeFor W et al. Antiphospholipid antibodies are a risk factor for early renal allograft failure. Transplantation 1999; 68:241-6. 
7. Ducloux D, Pellet E, Fournier V et al. Prevalence and clinical significance of antiphospholipid antibodies in renal transplant recipients. Transplantation 1999; 67:90-3.

8. Dowling N, Austin H, Dilley A et al. The epidemiology of venous thromboembolism in Caucasians and AfricanAmericans: the GATE Study. J Thromb Haemost 2003; 1:80-7.

9. Franco R, Fagundes M, Meijers J et al. Identification of polymorphisms in the 5'-untranslated region of the TAFI gene: relationship with plasma TAFI levels and risk of venous thrombosis. Haematologica 2001; 86:510-7.

10. Morelli V, Lourenço D, D'Almeida V et al. Hyperhomocysteinemia increases the risk of venous thrombosis independent of the C677T mutation of the methylenetetrahydrofolate reductase gene in selected Brazilian patients. Blood Coagul Fibrinolysis 2002; $13: 271-5$

11. Galli M, Borrelli G, Jacobsen E et al. Clinical significance of different antiphospholipid antibodies in the WAPS (warfarin in the antiphospholipid syndrome) study. Blood 2007; 110:1178-83.

12. Medina-Pestana J. Organization of a high-volume kidney transplant program - the "assembly line" approach. Transplantation 2006; 81:1510-20.

13. Gomez E, van der Poel S, Jansen J et al. Rapid simultaneous screening of factor V Leiden and G20210A prothrombin variant by multiplex polymerase chain reaction on whole blood. Blood 1998; 91:2208-9.

14. Bertina R, Rosendaal F. Venous thrombosis - the interaction of genes and environment. N Engl J Med 1998; 338:1840-1.

15. Rosenberg R, Aird W. Vascular-bed - specific hemostasis and hypercoagulable states. N Engl J Med 1999; 340:1555-64.

16. Petri M. Epidemiology of the antiphospholipid antibody syndrome. J Autoimmun 2000; 15:145-51.

17. Merkel P, Chang Y, Pierangeli S et al. The prevalence and clinical associations of anticardiolipin antibodies in a large inception cohort of patients with connective tissue diseases. Am J Med 1996; 101:576-83.

18. Vaidya S, Sellers R, Kimball P et al. Frequency, potential risk and therapeutic intervention in end-stage renal disease patients with antiphospholipid antibody syndrome: a multicenter study. Transplantation 2000; 69:1348-52.

19. Ginsburg K, Liang M, Newcomer Let al. Anticardiolipin antibodies and the risk for ischemic stroke and venous thrombosis. Ann Intern Med 1992; 117:997-1002.

20. Vaarala O, Mänttäri M, Manninen V et al. Anticardiolipin antibodies and risk of myocardial infarction in a prospective cohort of middle-aged men. Circulation $1995 ; 91: 23-7$.

21. Levine S, Brey R, Joseph C et al. Risk of recurrent thromboembolic events in patients with focal cerebral ischemia and antiphospholipid antibodies. The Antiphospholipid Antibodies in Stroke Study Group. Stroke 1992; 23:129-32.
22. McIntyre J, Wagenknecht D. Antiphospholipid antibodies and renal transplantation: a risk assessment. Lupus 2003; 12:555-9.

23. Pherwani A, Winter $\mathrm{P}, \mathrm{McNamee} \mathrm{P}$ et al. Is screening for factor V Leiden and prothrombin G20210A mutations in renal transplantation worthwhile? Results of a large single-center U.K. study. Transplantation 2003; 76:603-5.

24. Irish A, Green F, Gray D et al. The factor V Leiden (R506Q) mutation and risk of thrombosis in renal transplant recipients. Transplantation 1997; 64:604-7.

25. Ekberg H, Svensson P, Simanaitis M et al. Factor V R506Q mutation (activated protein $\mathrm{C}$ resistance) is an additional risk factor for early renal graft loss associated with acute vascular rejection. Transplantation 2000; 69:1577-81.

26. Irish A. Cardiovascular disease, fibrinogen and the acute phase response: associations with lipids and blood pressure in patients with chronic renal disease. Atherosclerosis 1998; 137:133-9.

27. Irish A, Green F. Factor VII coagulant activity (VIIc) and hypercoagulability in chronic renal disease and dialysis: relationship with dyslipidaemia, inflammation, and factor VII genotype. Nephrol Dial Transplant 1998; 13:679-84.

28. Walkowiak B, Pawlowska Z, Michalak E et al. Expression of fibrinogen receptors on platelets of uremic patients is correlated with the content of GPIIb and plasma level of creatinine. Thromb Haemost 1994; 71:164-8.

29. Baker L, Tucker B, Kovacs I. Enhanced in vitro hemostasis and reduced thrombolysis in cyclosporine-treated renal transplant recipients. Transplantation 1990; 49:905-9.

30. Brunkwall J, Bergqvist D, Almér L et al. Tissue plasminogen activator, its fast acting plasma inhibitor and protein $\mathrm{C}$ after renal transplantation. Thromb Res 1995; 77:105-11.

31. Carlesen E, Prydz H. Enhancement of procoagulant activity in stimulated mononuclear blood cells and monocytes by cyclosporine. Transplantation 1987; 43:543-8.

32. Díaz-Ricart M, Estebanell E, Cases A et al. Abnormal platelet cytoskeletal assembly in hemodialyzed patients results in deficient tyrosine phosphorylation signaling. Kidney Int 2000; 57:1905-14.

33. Matas A, Humar A, Gillingham K et al. Five preventable causes of kidney graft loss in the 1990s: a singlecenter analysis. Kidney Int 2002; 62:704-14.

34. Penny M, Nankivell B, Disney A et al. Renal graft thrombosis. A survey of 134 consecutive cases. Transplantation 1994; 58:565-9.

35. Bakir N, Sluiter W, Ploeg R et al. Primary renal graft thrombosis. Nephrol Dial Transplant 1996; 11:140-7.

36. Ojo A, Hanson J, Wolfe R et al. Dialysis modality and the risk of allograft thrombosis in adult renal transplant recipients. Kidney Int 1999; 55:1952-60. 